\title{
Comprehensive analysis of prognostic tumor microenvironment-related genes in osteosarcoma patients
}

\author{
Chuan Hu', Chuan Liu², Shaoqi Tian', Yuanhe Wang ${ }^{1}$, Rui Shen', Huili Rao ${ }^{3}$, Jianyi Li', Xu Yang ${ }^{1}$, Bo Chen ${ }^{4}$ and \\ $\operatorname{Lin} Y e^{4^{*}}$
}

\begin{abstract}
Background: Tumor microenvironment (TME) plays an important role in malignant tumors. Our study aimed to investigate the effect of the TME and related genes in osteosarcoma patients.

Methods: Gene expression profiles and clinical data of osteosarcoma patients were downloaded from the TARGET dataset. ESTIMATE algorithm was used to quantify the immune score. Then, the association between immune score and prognosis was studied. Afterward, a differential analysis was performed based on the high- and low-immune scores to determine TME-related genes. Additionally, Cox analyses were performed to construct two prognostic signatures for overall survival (OS) and disease-free survival (DFS), respectively. Two datasets obtained from the GEO database were used to validate signatures.

Results: Eighty-five patients were included in our research. The survival analysis indicated that patients with higher immune score have a favorable OS and DFS. Moreover, 769 genes were determined as TME-related genes. The unsupervised clustering analysis revealed two clusters were significantly related to immune score and T cells CD4 memory fraction. In addition, two signatures were generated based on three and two TME-related genes, respectively. Both two signatures can significantly divide patients into low- and high-risk groups and were validated in two GEO datasets. Afterward, the risk score and metastatic status were identified as independent prognostic factors for both OS and DFS and two nomograms were generated. The C-indexes of OS nomogram and DFS nomogram were 0.791 and 0.711 , respectively.
\end{abstract}

Conclusion: TME was associated with the prognosis of osteosarcoma patients. Prognostic models based on TMErelated genes can effectively predict OS and DFS of osteosarcoma patients.

Keywords: Tumor microenvironment, Osteosarcoma, Prognosis, Immune features, Nomogram

\section{Background}

Osteosarcoma is the most common bone tumor, especially in children and adolescents [1]. It was reported that approximately $60 \%$ of patients are between 10 and 20 years old and osteosarcoma is considered as the second leading cause of death in this age group [2]. Currently, surgery and

* Correspondence: 407404159@qq.com

${ }^{4}$ Wenzhou Medical University, Wenzhou, China

Full list of author information is available at the end of the article chemotherapy are still major treatments for osteosarcoma patients, and these therapies are constantly improving in recent years. However, due to the susceptibility of local aggressiveness and lung metastasis in osteosarcoma patients, the prognosis of osteosarcoma remains unfavorable [3]. Previous studies indicated that the 5-years survival rates were 27.4 and $70 \%$ in metastatic and non-metastatic patients, respectively [4]. Therefore, it is necessary to

(c) The Author(s). 2020 Open Access This article is licensed under a Creative Commons Attribution 4.0 International License, which permits use, sharing, adaptation, distribution and reproduction in any medium or format, as long as you give appropriate credit to the original author(s) and the source, provide a link to the Creative Commons licence, and indicate if changes were made. The images or other third party material in this article are included in the article's Creative Commons licence, unless indicated otherwise in a credit line to the material. If material is not included in the article's Creative Commons licence and your intended use is not permitted by statutory regulation or exceeds the permitted use, you will need to obtain permission directly from the copyright holder. To view a copy of this licence, visit http://creativecommons.org/licenses/by/4.0/ The Creative Commons Public Domain Dedication waiver (http://creativecommons.org/publicdomain/zero/1.0/) applies to the data made available in this article, unless otherwise stated in a credit line to the data. 
investigate the mechanism of pathogenesis and progression of osteosarcoma and accurately classify the risk of patients.

Recently, an increasing number of diagnostic and prognostic biomarkers of osteosarcoma patients have been identified. For example, Chen et al. [5] reported that tumor suppressor p27 is a novel biomarker for the metastasis and survival status in osteosarcoma patients. Moreover, Huang et al. [6] discovered that dysregulated circRNAs serve as prognostic and diagnostic biomarkers in osteosarcoma patients, and the relative potential mechanism mainly attributes to the regulation of downstream signaling pathways by sponging microRNA. In addition, IncRNA [7], microRNA [8], and many clinical data [9] were also identified as prognostic biomarkers for osteosarcoma patients. However, osteosarcoma is one of the malignant cancers entities characterized by the high level of heterogeneity in humans. Therefore, it is necessary to find accurate biomarkers for osteosarcoma.

In recent years, researchers have paid more and more attention to the role of the tumor microenvironment (TME) in malignant tumors. The function of TME in the tumorigenesis, progression, and therapy of tumors have been initially understood $[10,11]$. More importantly, Estimation of STromal and Immune cells in MAlignant Tumor tissues using Expression data (ESTIMA $\mathrm{TE})$, an algorithm to quantify the score of immune cells and stromal cells by analyzing the gene expression data, was developed in 2013 [12]. Based on the algorithm, the prognostic value of immune and stromal cells in bladder cancer, acute myeloid leukemia, gastric cancer, cervical squamous cell carcinoma, adrenocortical carcinoma, clear cell renal cell carcinoma, hepatocellular carcinoma, thyroid cancer, and cutaneous melanoma have been reported [13-23]. Generally, the above research indicated that TME can serve as the prognostic biomarker in tumors, and many TME-related genes were determined as the prognostic genes. However, the role of TME and TME-related genes in osteosarcoma patients remains unclear.

In the present study, gene expression data and corresponding clinicopathologic data were obtained from The Therapeutically Applicable Research to Generate Effective Treatments (TARGET) dataset. Then, the ESTIMATE algorithm was performed to quantify the immune score of osteosarcoma and the TME-related genes were identified by the differential expression analysis. Subsequently, the prognostic value of TME and TME-related genes were determined by a series of bioinformatics methods.

\section{Methods}

\section{Gene expression datasets}

Level 3 data of gene expression profiles and corresponding clinical data of osteosarcoma patients were downloaded from TARGET dataset (https://ocg.cancer.gov/programs/ target, accessed on Oct 11, 2019). The corresponding clinicopathologic data included in the present study were age, gender, race, ethnicity, tumor site, and metastatic status. After data were extracted from the public domain, the ESTIMATE, an algorithm inferring tumor purity, stromal score, and immune cell admixture from expression data, was performed to evaluate the immune score by using the estimate package in $\mathrm{R}$ software (version 3.6.1) [12]. Meanwhile, the messenger RNA(mRNA) expression profiles and clinical data of two cohorts, including GSE21257 [24] and GSE39055 [25], were obtained from the Gene Expression Omnibus as external validation cohorts.

\section{Survival analysis and correlation analysis}

After scores were obtained, patients were divided into high-score group and low-score group according to the median of the immune score. The Kaplan-Meier survival analysis with log-rank test was performed to estimate the differences of overall survival (OS) and disease-free survival (DFS) between high- and low-score cohorts. In addition, the association between clinicopathologic data and TME score was also studied. Mann-Whitney signedrank test was performed to compare the differences of immune score between each clinical group. All statistical analyses in the present study were performed using $\mathrm{R}$ software. Except for the special instructions, $p$ value $<$ 0.05 (two-side) was identified as statistically significant in the present study.

\section{Differentially expressed gene analysis}

Differentially expressed gene (DEG) analysis was performed by comparing the protein-coding genes expression between the low-immune score group and the high-immune score group. The limma package in $\mathrm{R}$ software was used to perform the differential analysis and genes with $|\log \mathrm{FC}|>1.0$ and adjusted $p$-value (q value) $<0.05$ were identified as DEGs [26].

To further understand the function of DEGs identified in the present study, Gene Ontology (GO), including biological processes (BP), molecular functions (MF), and cellular components(CC) and Kyoto Encyclopedia of Genes and Genomes (KEGG) analysis were performed by clusterProfiler package in R software [27].

\section{Evaluation of association with immune cells}

To further investigate the association between DEGs and immune cells, the CIBERSORT package was used to estimate the relative proportions of 22 types of immune cells [28]. Meanwhile, the "ConsensusClusterPlus" package was used to cluster in an unbiased and unsupervised manner based on the overlapping DEGs [29]. Cumulative distribution function (CDF) and relative change in area under the CDF curve were used to determine the optimal number of clusters k. Then, Mann-Whitney 
signed-rank test was performed to study the difference of immune cells proportion between the clusters and the violin plot was established to show the differences of immune cells among clusters [30].

\section{Survival analysis of DEGs}

Based on the DEGs, the univariate COX analysis was performed to determine the prognostic value of immunerelated genes. Then, the OS-related genes were validated in the GSE21257 dataset, while the DFS-related genes were validated in the GSE39055 dataset. Only genes successfully validated were selected for further analysis. Afterward, based on the validated genes, the multivariate COX analysis was performed to establish the prognostic signature for predicting the prognosis of osteosarcoma patients. The risk score for each patient was calculated based on the coefficient from the multivariate COX analysis and the corresponding gene expression. Meanwhile, all patients were divided into the high- and low-risk groups according to the median of the risk score. The survival receiver operating characteristic (ROC) curve was used to show the discrimination of signatures, and the Kaplan-Meier survival curve with the log-rank test was generated to show the differences of OS and DFS between high- and low-risk groups. In addition, the risk score of patients in the validation cohort was also calculated according to the aforementioned risk signature. The Kaplan-Meier survival curve and survival ROC curve were generated to show the predictive ability of the signature in the validation cohort.

\section{Development of a nomogram for osteosarcoma patients}

Nomogram is a tool to visualize the predictive model and convenient for clinical practice. Therefore, we attempted to develop a nomogram based on the TME-related genes signature and clinicopathologic data to predict the prognosis of osteosarcoma patients. Firstly, the univariate
COX analysis was performed to filter prognostic variables, which will be further included in the multivariate COX analysis. Secondly, based on independent prognostic variables, two nomograms were established for predicting the OS and DFS, respectively. The C-index was used to assess the discriminatory performance of the nomogram, which range from 0.5 to 1 [31]. A C-index of 0.5 means agreement by chance and a C-index of 1 represents perfect discriminatory performance. The higher value of the $\mathrm{C}$ index, the better performance of the nomogram is. Furthermore, the calibration curves of 1-, 2-, and 3-year were developed to evaluate the effectiveness of nomograms.

\section{Results}

Immune significantly associated with the prognosis of osteosarcoma patients

85 osteosarcoma patients were included in the present study, including 48 males and 37 females. The immune score of the cohort range from -1459.56 to 2581.96 . To study the relationship between the immune score and the prognosis of osteosarcoma patients, 42 patients were incorporated into the low-immune score group, while the remaining 43 patients were incorporated into the highimmune score group. The survival analysis indicated that patients with higher immune score had a favorable OS and DFS (Fig. 1a and b). After adjusted age, tumor site, and metastatic status, the immune score still was a prognostic variable for both OS and DFS(Fig. 1a and b). In addition, the relationship between immune score and clinical features was also investigated. However, there was no significant relationship between immune score and clinical variables (Supplementary Figure 1A-1C).

\section{Differential expression analysis}

According to the median of the immune score, 85 patients were divided into high-score $(n=43)$ and low-
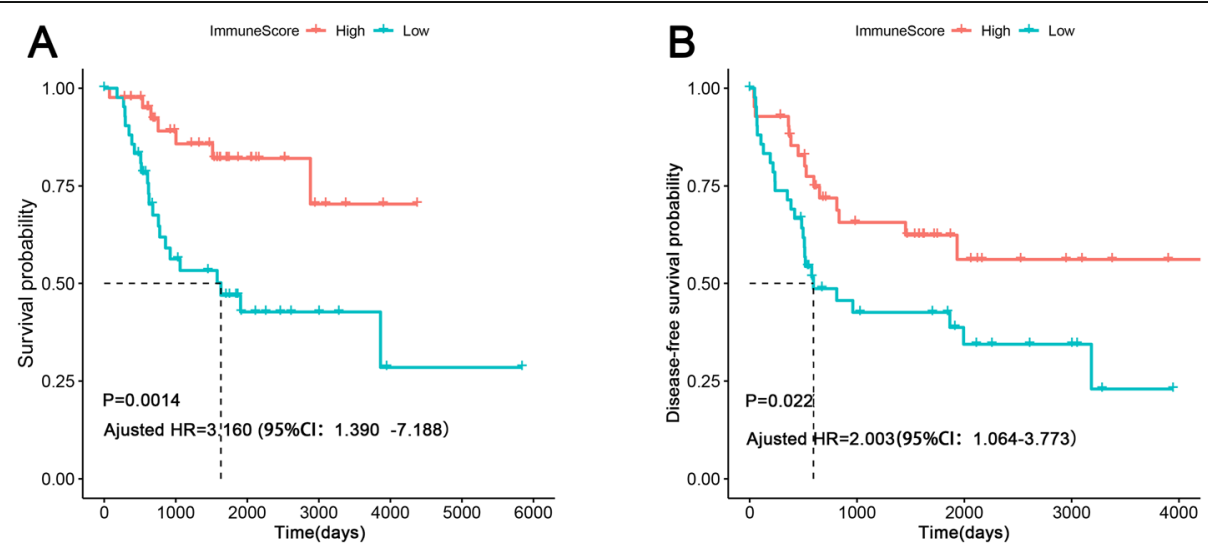

Fig. 1 Association between immune score and prognosis in osteosarcoma patients. a Kaplan-Meier survival analysis of overall survival for patients with high vs. low immune score; b Kaplan-Meier survival analysis of disease-free survival for patients with high vs. low immune score 
score group $(n=42)$. There were 769 differentially expressed genes between two groups, which include 498 upregulated genes and 271 downregulated genes (Fig. 2a, b, and Supplementary Table 1). To further understand the function of 769 DEGs, GO analysis and KEGG analysis were performed. The top 10 significant results of $\mathrm{GO}$ analysis among three types were illustrated in Fig. 2c. Interestingly, we can find that the results of GO analysis are mostly associated with immunity, which further verify that the immune-related DEGs are associated with immune features. In addition, the results of KEGG also confirmed it. Such as "Phagosome", "Autoimmune thyroid disease", "Antigen processing and presentation", "B cell receptor signaling pathway", "Intestinal immune network for IgA production", "Inflammatory bowel disease", "Primary immunodeficiency", "Th1 and Th2 cell differentiation", "Th17 cell differentiation", "Natural killer cell mediated cytotoxicity", and "NF-kappa B signaling pathway" (Fig. 2d).

\section{Evaluation of DEGs and immune cells}

To further understand the molecular heterogeneity of osteosarcoma, unsupervised consensus analysis was performed to divide patients into subgroups to explore whether immune-related genes presented discernable patterns. Based on the consensus matrix heat map, patients were clearly divided into two clusters(Fig. 3a). In addition, by comprehensively analyzing the relative change in area under the cumulative distribution function, two clusters were determined (Fig. 3b-c). The immune score between two clusters was significantly different (Fig. 3d). In addition, the proportion of 22 types of immune cells in osteosarcoma patients was illustrated in a barplot (Fig. 3e). Interestingly, we can see that the $\mathrm{T}$ cells $\mathrm{CD} 4$ memory activated of cluster 2 is significantly higher than cluster 1 (Fig. 5f).

\section{Prognostic value of TME-related genes}

Previous studies indicated that TME-related genes can serve as the prognostic biomarker for tumor patients.
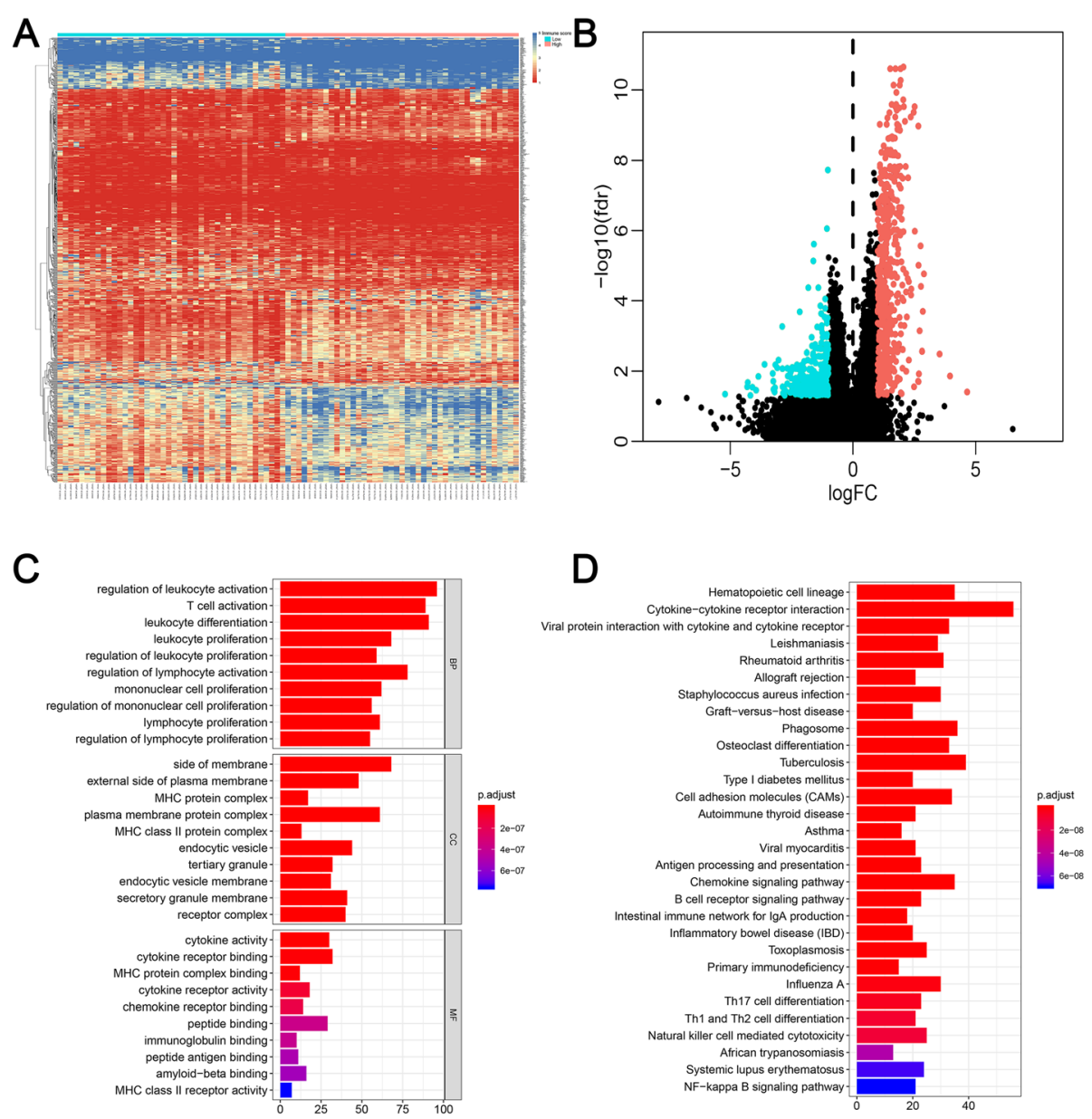

Fig. 2 Differentially expressed genes with the immune score in osteosarcoma patients. a Heatmap of significantly differentially expressed genes based on immune score; $\mathbf{b}$ The volcano figure to show the upregulated and downregulated genes. $\mathbf{c} G \mathrm{G}$ analysis of differentially expressed genes. d KEGG of differentially expressed genes. GO: Gene Ontology; KEGG: Kyoto Encyclopedia of Genes and Genomes 


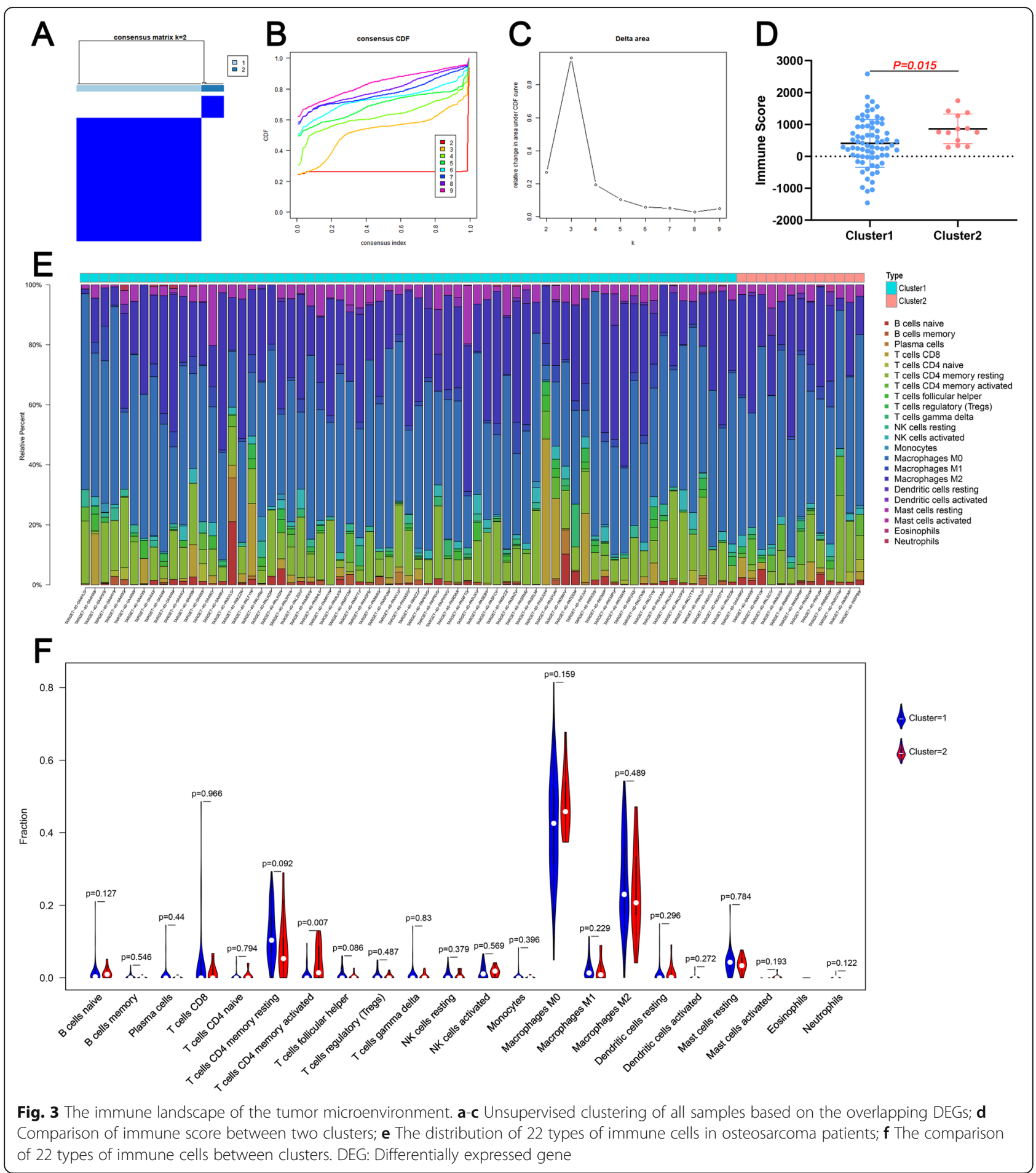

Hence, we performed the univariate COX analysis to identify prognostic DEGs. The results showed that 160 and 120 genes were identified as OS- and DFS-related DEGs, respectively (Supplementary Table 2 and 3). Afterward, five OS-related genes were successfully validated in the GSE21257 data set, and five DFS-related genes were successfully validated in the GSE39055 cohort. Furthermore, multivariate COX analysis was performed and two prognostic signatures were generated for predicting the OS and DFS, respectively. The risk score for predicting the OS was as follows: risk score $=$ FCGR2B $* 0.766+$ GFAP $* 0.702+$ MPP7*0.387. In addition, the risk score for predicting the DFS was as follows: risk score $=$ CYP2S1 ${ }^{*}-0.574+$ ICAM3 $^{*}-$ 2.015. The AUC values of OS-related signature were 0.811 , 
0.730, and 0.720 in 1-, 2-, and 3-year, respectively (Fig. 4a), and the AUC values of DFS-related signature were 0.690, 0.616, and 0.652 in 1-, 2-, and 3-year, respectively (Fig. 5a). Moreover, survival curves showed that patients in the highrisk group had worse OS and DFS compared with the lowrisk patients (Figs. 4b and 5b). Heat maps, risk score plots, and survival status were generated to show the distinction between high-risk patients and low-risk patients (Figs. 4c-e and $5 \mathrm{c}-\mathrm{e})$. Then, both signatures were validated in independent cohorts. For OS signature, the AUC values of validation cohort were $0.811,0.750$, and 0.723 at $1-, 2-$, and 3-year (Fig. 4f). For DFS signature, the AUC values of validation cohort were $0.856,0.683$, and 0.770 at 1-, 2-, and 3-year (Fig. 5f). Additionally, in both validation cohorts, survival curves showed that low-risk patients were favorable prognosis than high-risk patients (Figs. $4 \mathrm{~g}$ and $5 \mathrm{~g}$ ). Heat maps, risk score plots, and survival status of validation cohorts were also generated to show the distinction between high-risk patients and low-risk patients (Figs. $4 \mathrm{~h}-\mathrm{j}$ and $\mathrm{f} 5 \mathrm{~h}-\mathrm{j}$ ).

\section{Development of a nomogram for osteosarcoma patients}

To generate a nomogram for clinical use, the COX analysis was performed to select the clinical prognostic variables. In

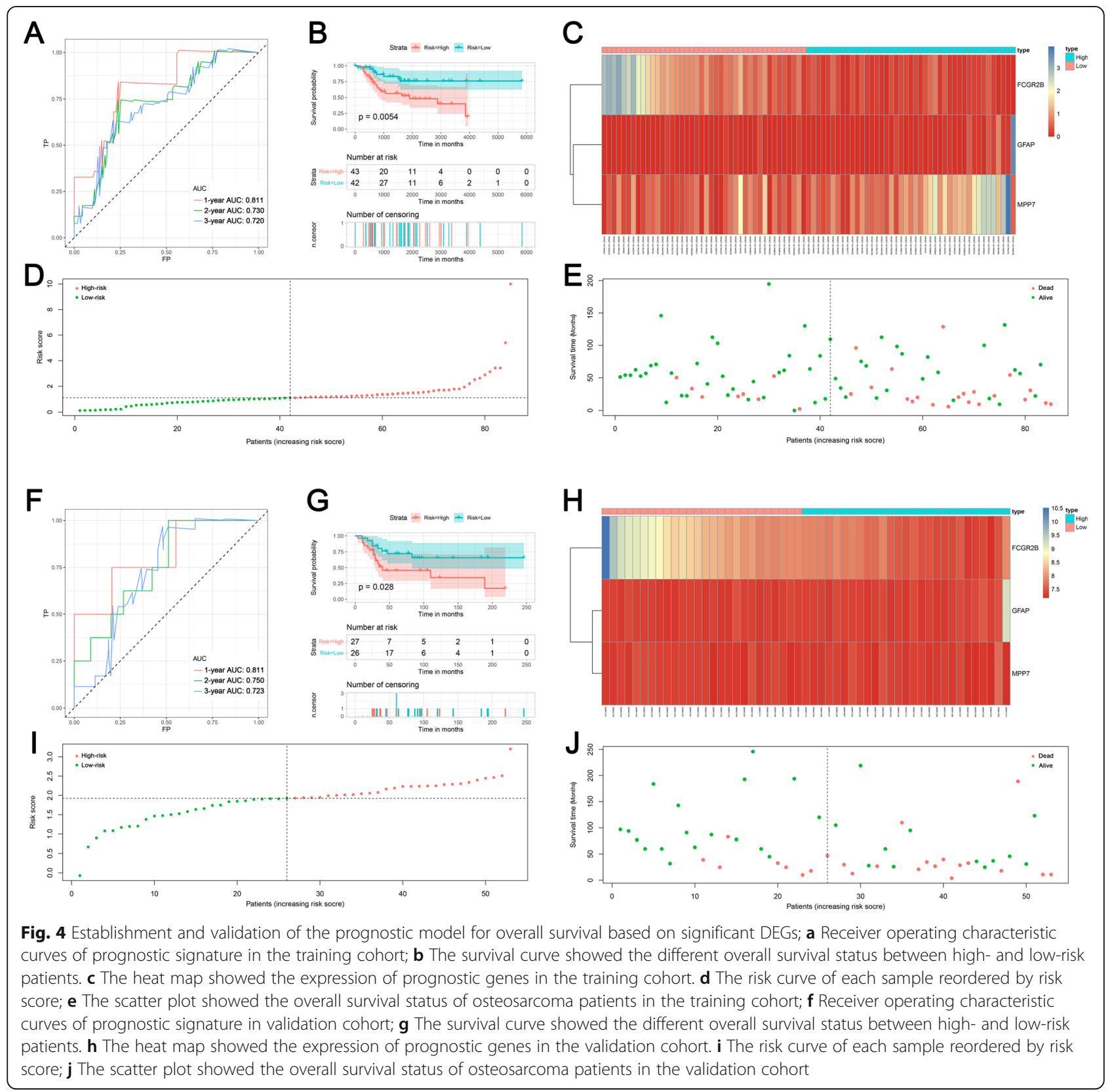




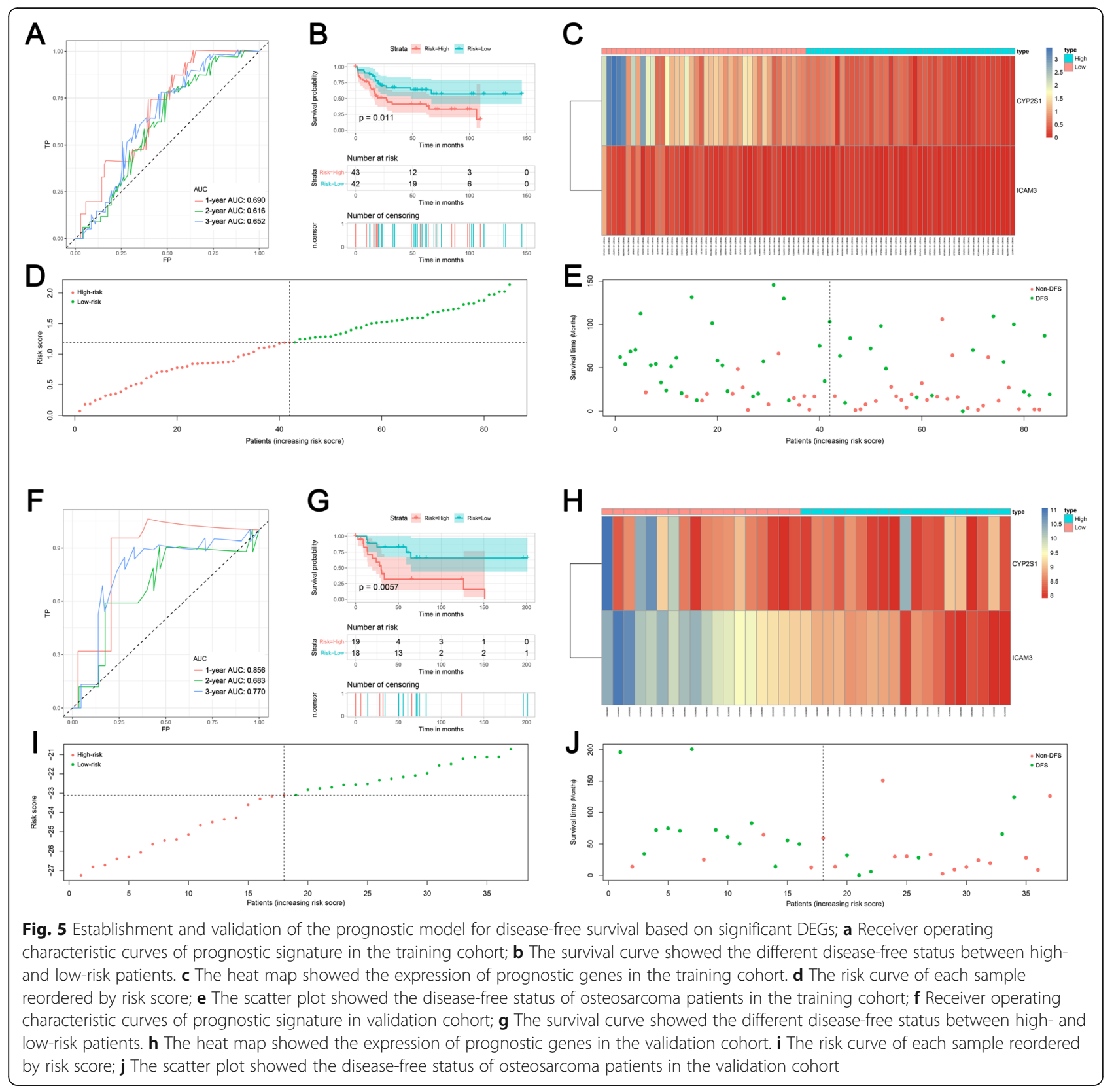

the univariate COX analysis, risk score and metastatic status were identified as both OS- and DFS-related variables (Fig. 6a and e). Afterward, risk score and metastatic status were determined as both independent OS- and DFSrelated variables in the multivariate COX analysis (Fig. 6b and $\mathrm{f}$ ). Based on independent variables, two nomograms were established for predicting the OS and DFS in osteosarcoma patients, respectively (Fig. $6 \mathrm{c}$ and g). The C-index values were 0.739 and 0.687 in OS nomogram and DFS nomogram, respectively. The results of $\mathrm{C}$-index mean that both two nomograms have good discrimination. Meanwhile, to evaluate the calibration of nomograms, six calibration curves were generated and the results showed that the predictive curves were close to the ideal curve (Fig. 6d and $\mathrm{h}$ ), which indicated a good calibration.

\section{Discussion}

The relationship between TME and tumor have been widely studied in recent years. In the present study, ESTI MATE algorithm was utilized to quantify the immune score based on gene expression profiles in 85 osteosarcoma patients from TARGET database. We confirmed that the TME is significantly associated with the prognosis of osteosarcoma patients, including OS and DFS. In addition, functional enrichment analyses of TMErelated genes indicated that immune-related processes 


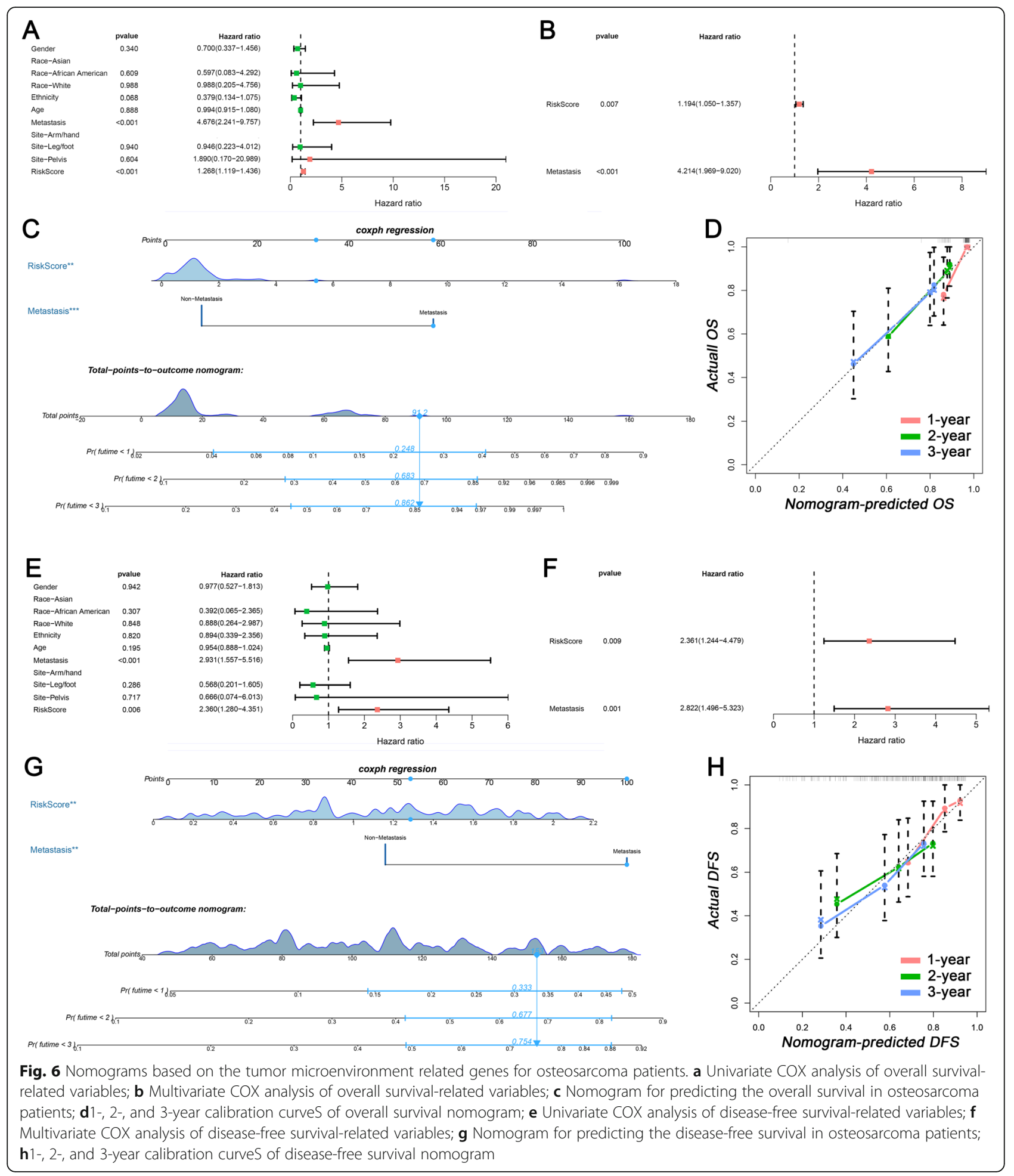

known to contribute to tumor progression. More importantly, DEGs based on the TME were identified as important prognostic biomarkers for osteosarcoma patients, and two nomograms were developed for predicting the OS and DFS of osteosarcoma patients, respectively.
In recent years, an increasing number of studies focused on the carcinogenesis and progression of tumors based on the TME, and the ESTIMATE algorithm is one of the most important quantitative tools for this research field. Based on the ESTIMATE algorithm, the association between the prognosis and TME has been initially 
elucidated in some tumors, such as cervical squamous cell carcinoma, gastric cancer, cutaneous melanoma, acute myeloid leukemia, bladder cancer, and clear cell renal carcinoma [13, 16, 17, 19-23]. However, previous studies indicated that TME scores serve as a different role in different tumors. For example, for hepatocellular carcinoma, gastric cancer, acute myeloid leukemia, bladder cancer, and clear cell renal carcinoma, patients with high immune score have a worse prognosis $[14,16$, 17, 20-23]. However, for cervical squamous cell carcinoma, adrenocortical carcinoma, and cutaneous melanoma, patients with high immune score have a favorable prognosis $[13,18,19]$. Therefore, we can find great heterogeneity among different tumors from the perspective of TME. For osteosarcoma patients, the present study indicated that patients with higher immune score had a better OS and DFS. Hence, the present study indicated that immune cells infiltrating tumor tissue may play an important role in suppressing tumor progression.

In our research, 769 TME-related genes were identified by comparing the high-score and low-score osteosarcoma patients. The functional enrichment, including GO and KEGG analyses, showed that TME-related genes were mainly involved in the immune features, such as regulation of leukocyte activation, MHC protein complex, MHC protein, and complex binding. More importantly, the unsupervised cluster analysis based on DEGs was performed and all patients were divided into two clusters. Immune score and T cell CD4 memory activated fraction were significant difference between two clusters, which further elucidated the relationship between DEGs and immune features.

Due to the poor prognosis of osteosarcoma patients, identifying robust prognostic biomarker is very important. The tumor immune microenvironment is closely related to the prognosis of bone tumor patients. Emilie et.al [24] performed the first genome-wide study to describe the role of immune cells in osteosarcoma and found that tumor-associated macrophages are associated with reduced metastasis and improved survival in high-grade osteosarcoma. Recently, the prognostic signature based on TME-related genes have been established for many tumors $[18,20,32]$, but only one study focused on osteosarcoma patients [33]. Compared with the study performed by Zhang et al. [33], we think that our research have some advantages. Firstly, our signatures were established based on several validated genes, and both two signatures were successfully validated in independent cohorts. Secondly, the outcome of DFS was not reported in the previous study. As reported in published studies, tumor recurrence is a terrible medical problem for osteosarcoma patients, and the 5-year survival rate for osteosarcoma patients with metastasis or relapse remains disappointing [34, 35]. Hence, the DFS nomogram can improve the management of osteosarcoma patients. Finally, two nomograms incorporated TME-related signature and clinical variables were established in our research, which further facilitated the clinical application of our findings.

In our research, five genes were incorporated into the final prognostic signatures. FCGR2B, GFAP, and MPP7 were identified and validated as OS-related biomarkers, while CYP2S1 and ICAM3 were DFS-related biomarkers. The role of these genes in tumor prognosis had been widely reported in previous studies [36-40]. FCGR2B has been confirmed as an immune-related gene previously [41]. Although the relationship between FCGR2B and prognosis in sarcoma patients had not been reported, the prognostic value of FCGR2B had been widely confirmed in other cancers, such as hepatocellular carcinoma and glioblastoma [36, 42]. In addition, New $\mathrm{M}$ et.al [37] demonstrated that MPP7 is novel regulators of autophagy, which was thought to be responsible for the prognosis of pancreatic ductal adenocarcinoma. CYP2S1, described as Cytochrome P450 Family 2 Subfamily $S$ Member 1, was reported significantly associated with colorectal cancer. In primary colorectal cancer, CYP2S1 was present at a significantly higher level of intensity compared with normal colon [43]. More importantly, the presence of strong CYP2S1 immunoreactivity was associated with poor prognosis [43]. The role of ICAM3 in cancer was also widely reported in published studies, and the Akt pathway plays an important role in the impact of ICAM3 on tumors. YG Kim et.al [44] reported that ICAM3 can induce the proliferation of cancer cells through the PI3K/Akt pathway. Additionally, JK Park et.al showed that the ICAM3 can enhance the migratory and invasive potential of human nonsmall cell lung cancer cells by inducing MMP-2 and MMP-9 via Akt pathway [45] showed that the ICAM3 can enhance the migratory and invasive potential of human non-small cell lung cancer cells by inducing MMP-2 and MMP-9 via Akt pathway.

Although the role of TME and TME-related genes in osteosarcoma patients have been initially studied by bioinformatic and statistical analyses in our research, some limitations should be elucidated. Firstly, the treatment information cannot be obtained from the TARGET database, which may influence the prognosis of osteosarcoma patients. Secondly, two nomograms were generated and showed good performance in our study. However, external validation by a large cohort is needed. Thirdly, many independent prognostic genes for osteosarcoma patients were identified in the present study, but the potential mechanism to influence osteosarcoma remains unclear. Finally, in the training cohort, 160 and 120 DEGs were identified as OS- and DFS-related DEGs, respectively. However, only five OS- and five DFS-related genes were identified in the validation cohort. The different age structures, smaller 
sample sizes and the platform covering only part of the genes may contribute to this result.

\section{Conclusion}

In conclusion, TME plays an important role in osteosarcoma patients and related with the progression of the tumor. Moreover, TME-related genes can serve as prognostic biomarkers in osteosarcoma patients. However, further researches are needed to study the potential mechanism and validate the nomogram that developed in our present study.

\section{Supplementary information}

Supplementary information accompanies this paper at https://doi.org/10. 1186/s12885-020-07216-2.

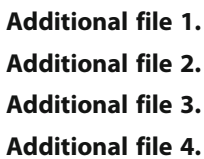

\section{Abbreviations}

TME: Tumor microenvironment; DEG: Differentially expressed genes; OS: Overall survival; DFS: Diseases-free survival; ROC: Receiver characteristic curve; ESTIMATE: Estimation of STromal and Immune cells in MAlignant Tumor tissues using Expression data; TARGET: Therapeutically Applicable Research to Generate Effective Treatments; GO: Gene Ontology; BP: Biological processes; MF: Molecular functions; CC: Cellular components; KEGG: Kyoto Encyclopedia of Genes and Genomes; CDF: Cumulative distribution function

\section{Acknowledgements}

None.

\section{Authors' contributions}

$C H, L Y, S q T, C L$ and $Y h W$ conceived of and designed the study. C H, R S and $C L$ performed literature search. $R$ S, $L Y$ and $B C$ generated the figures and tables. $L Y, H I R, X Y$ and Jy $L$ analyzed the data. $C H$ wrote the manuscript and $\mathrm{Sq} T$ and $L Y$ critically reviewed the manuscript. $L Y$ supervised the research. All authors have read and approved the manuscript.

\section{Funding}

We received no external funding for this study.

\section{Availability of data and materials}

The data of this study are from TARGET and GEO database.

\section{Ethics approval and consent to participate}

The research didn't involve animal experiments and human specimens, no ethics related issues.

\section{Consent for publication \\ Not applicable.}

\section{Competing interests}

The authors declare that they have no competing interests.

\section{Author details}

${ }^{1}$ Department of Joint Surgery, the Affiliated Hospital of Qingdao University, Qingdao, China. ${ }^{2}$ Department of Medical Oncology, the First Hospital of China Medical University, Shenyang, China. ${ }^{3}$ Department of Nursing, Sir Run Run Shaw Hospital Affiliated to Zhejiang University, Hangzhou, China. ${ }^{4}$ Wenzhou Medical University, Wenzhou, China.
Received: 3 February 2020 Accepted: 26 July 2020

Published online: 27 August 2020

\section{References}

1. Jaffe N, Bruland OS, Bielack S. Pediatric and adolescent osteosarcoma, vol. 152. New York: Springer Science \& Business Media; 2010.

2. Vander RG. Osteosarcoma and its variants. Orthopedic Clin North Am. 1996; 27(3):575-81.

3. Biermann JS, Adkins D, Benjamin R, Brigman B, Chow W, Conrad EU 3rd, Frassica D, Frassica FJ, George S, Healey JH, et al. Bone cancer. J Natl Compr Cancer Netw. 2007:5(4):420-37.

4. Simpson S, Dunning MD, de Brot S, Grau-Roma L, Mongan NP, Rutland CS. Comparative review of human and canine osteosarcoma: morphology, epidemiology, prognosis, treatment and genetics. Acta Vet Scand. 2017:59(1):71.

5. Chen X, Cates JM, Du Y-C, Jain A, Jung SY, Li X-N, Hicks JM, Man TK. Mislocalized cytoplasmic p27 activates PAK1-mediated metastasis and is a prognostic factor in osteosarcoma. Mol Oncol. 2020;14(4):846-64.

6. Huang X, Yang W, Zhang Z, Shao Z. Dysregulated circRNAs serve as prognostic and diagnostic markers in osteosarcoma by sponging microRNA to regulate the downstream signaling pathway. J Cell Biochem. 2019;121(2):1834-41.

7. Liu M, Yang P, Mao G, Deng J, Peng G, Ning X, Yang H, Sun H. Long noncoding RNA MALAT1 as a valuable biomarker for prognosis in osteosarcoma: a systematic review and meta-analysis. Int J Surg. 2019;72:206-13.

8. Xu K, Xiong W, Zhao S, Wang B. MicroRNA-106b serves as a prognostic biomarker and is associated with cell proliferation, migration, and invasion in osteosarcoma. Oncol Lett. 2019:18(3):3342-8.

9. Zheng W, Huang Y, Chen H, Wang N, Xiao W, Liang Y, Jiang X, Su W, Wen S. Nomogram application to predict overall and cancer-specific survival in osteosarcoma. Cancer Manag Res. 2018;10:5439.

10. Kahlert C, Kalluri R. Exosomes in tumor microenvironment influence cancer progression and metastasis. J Mol Med. 2013;91(4):431-7.

11. Binnewies $M$, Roberts EW, Kersten $K$, Chan V, Fearon DF, Merad M, Coussens LM, Gabrilovich DI, Ostrand-Rosenberg S, Hedrick CC. Understanding the tumor immune microenvironment (TIME) for effective therapy. Nat Med. 2018;24(5):541-50

12. Yoshihara K, Shahmoradgoli M, Martínez E, Vegesna R, Kim H, Torres-Garcia W, Treviño V, Shen H, Laird PW, Levine DA. Inferring tumour purity and stromal and immune cell admixture from expression data. Nat Commun. 2013;4:2612.

13. Yang $S$, Liu T, Nan H, Wang Y, Chen H, Zhang X, Zhang Y, Shen B, Qian P, $X u S$, et al. Comprehensive analysis of prognostic immune-related genes in the tumor microenvironment of cutaneous melanoma. J Cell Physiol. 2020; 235(2):1025-35.

14. Deng Z, Wang J, Xu B, Jin Z, Wu G, Zeng J, Peng M, Guo Y, Wen Z. Mining TCGA database for tumor microenvironment-related genes of prognostic value in hepatocellular carcinoma. Biomed Res Int. 2019;2019:2408348.

15. Zhao K, Yang H, Kang H, Wu A. Identification of key genes in thyroid Cancer microenvironment. Med Sci Monit. 2019;25:9602

16. Xu W-H, Xu Y, Wang J, Wan F-N, Wang H-K, Cao D-L, Shi G-H, Qu Y-Y, Zhang $\mathrm{H}-\mathrm{L}$, Ye D-W. Prognostic value and immune infiltration of novel signatures in clear cell renal cell carcinoma microenvironment. Aging (Albany NY). 2019:11(17):6999.

17. Chen B, Chen W, Jin J, Wang X, Cao Y, He Y. Data Mining of Prognostic Microenvironment-Related Genes in clear cell renal cell carcinoma: a study with TCGA database. Dis Markers. 2019;2019:8901649.

18. Li X, Gao Y, Xu Z, Zhang Z, Zheng Y, Qi F. Identification of prognostic genes in adrenocortical carcinoma microenvironment based on bioinformatic methods. Cancer Med. 2019;9(3):1161-72.

19. Pan $X-B$, Lu Y, Huang J-L, Long Y, Yao D-S. Prognostic genes in the tumor microenvironment in cervical squamous cell carcinoma. Aging (Albany NY). 2019:11(22):10154

20. Wang H, Wu X, Chen Y. Stromal-immune score-based gene signature: a prognosis stratification tool in gastric Cancer. Front Oncol. 2019:9:1212.

21. Huang S, Zhang B, Fan W, Zhao Q, Yang L, Xin W, Fu D. Identification of prognostic genes in the acute myeloid leukemia microenvironment. Aging (Albany NY). 2019;11(22):10557.

22. Yan H, Qu J, Cao W, Liu Y, Zheng G, Zhang E, Cai Z. Identification of prognostic genes in the acute myeloid leukemia immune microenvironment based on TCGA data analysis. Cancer Immunol Immunother. 2019;68(12):1971-8. 
23. Luo Y, Zeng G, Wu S. Identification of microenvironment-related prognostic genes in bladder Cancer based on gene expression profile. Front Genet. 2019;10:1187.

24. Buddingh EP, Kuijjer ML, Duim RA, Bürger H, Agelopoulos K, Myklebost O, Serra M, Mertens F, Hogendoorn PC, Lankester AC, et al. Tumor-infiltrating macrophages are associated with metastasis suppression in high-grade osteosarcoma: a rationale for treatment with macrophage activating agents. Clin Cancer Res. 2011;17(8):2110-9.

25. Kelly AD, Haibe-Kains B, Janeway KA, Hill KE, Howe E, Goldsmith J, Kurek K, Perez-Atayde AR, Francoeur N, Fan JB, et al. MicroRNA paraffin-based studies in osteosarcoma reveal reproducible independent prognostic profiles at 14q32. Genome medicine. 2013;5(1):2.

26. Smyth GK. Limma: linear models for microarray data. In: Bioinformatics and computational biology solutions using $\mathrm{R}$ and Bioconductor. New York: Springer; 2005. p. 397-420.

27. Yu G, Wang L-G, Han Y, He Q-Y. clusterProfiler: an R package for comparing biological themes among gene clusters. OMICS. 2012;16(5):284-7.

28. Newman AM, Liu CL, Green MR, Gentles AJ, Feng W, Xu Y, Hoang CD, Diehn M, Alizadeh AA. Robust enumeration of cell subsets from tissue expression profiles. Nat Methods. 2015;12(5):453.

29. Wilkerson M, Hayes D. ConsensusClusterPlus: A class discovery tool with confidence assessments and item tracking. Bioinformatics (Oxford, England). 2010;26:1572-3.

30. Hintze JL, Nelson RD. Violin plots: a box plot-density trace synergism. Am Stat. 1998;52(2):181-4

31. Harrell FE Jr, Lee KL, Mark DB. Multivariable prognostic models: issues in developing models, evaluating assumptions and adequacy, and measuring and reducing errors. Stat Med. 1996;15(4):361-87.

32. Wang Z, Zhu J, Liu Y, Liu C, Wang W, Chen F, Ma L. Development and validation of a novel immune-related prognostic model in hepatocellular carcinoma. J Transl Med. 2020;18(1):67.

33. Zhang C, Zheng J-H, Lin Z-H, Lv H-Y, Ye Z-M, Chen Y-P, Zhang X-Y. Profiles of immune cell infiltration and immune-related genes in the tumor microenvironment of osteosarcoma. Aging (Albany NY). 2020;12(4):3486

34. Kager L, Zoubek A, Potschger U, Kastner U, Flege S, Kempf-Bielack B, Branscheid D, Kotz R, Salzer-Kuntschik M, Winkelmann W, et al. Primary metastatic osteosarcoma: presentation and outcome of patients treated on neoadjuvant cooperative osteosarcoma study group protocols. J Clin Oncol. 2003;21(10):2011-8.

35. Mirabello L, Troisi RJ, Savage SA. International osteosarcoma incidence patterns in children and adolescents, middle ages and elderly persons. Int J Cancer. 2009;125(1):229-34

36. Hu B, Yang X-B, Sang X-T. Development of an immune-related prognostic index associated with hepatocellular carcinoma. Aging (Albany NY). 2020;12(6):5010.

37. New M, Van Acker T, Sakamaki J-I, Jiang M, Saunders RE, Long J, Wang VM Y, Behrens A, Cerveira J, Sudhakar P. MDH1 and MPP7 regulate autophagy in pancreatic ductal adenocarcinoma. Cancer Res. 2019;79(8):1884-98.

38. Yang C, Zhou Q, Li M, Tong X, Sun J, Qing Y, Sun L, Yang X, Hu X, Jiang J. Upregulation of CYP2S1 by oxaliplatin is associated with p53 status in colorectal cancer cell lines. Sci Rep. 2016;6(1):1-11.

39. Liu X, Cao Y, Li R, Gu Y, Chen Y, Qi Y, Lv K, Wang J, Yu K, Lin C. Poor clinical outcomes of intratumoral dendritic cell-specific intercellular adhesion molecule 3-grabbing non-integrin-positive macrophages associated with immune evasion in gastric cancer. Eur J Cancer. 2020;128:27-37.

40. Ahmadipour $Y$, Gembruch O, Pierscianek D, Sure U, Jabbarli R. Does the expression of glial fibrillary acid protein (GFAP) stain in glioblastoma tissue have a prognostic impact on survival? Neurochirurgie. 2020;66(3):150-4.

41. Bhattacharya S, Andorf S, Gomes L, Dunn P, Schaefer H, Pontius J, Berger P, Desborough V, Smith T, Campbell J. ImmPort: disseminating data to the public for the future of immunology. Immunol Res. 2014;58(2-3):234-9.

42. Cheng W, Ren X, Zhang C, Cai J, Liu Y, Han S, Wu A. Bioinformatic profiling identifies an immune-related risk signature for glioblastoma. Neurology. 2016;86(24):2226-34

43. Kumarakulasingham M, Rooney PH, Dundas SR, Telfer C, Melvin WT, Curran S, Murray Gl. Cytochrome P450 profile of colorectal Cancer: identification of markers of prognosis. Clin Cancer Res. 2005;11(10):3758-65.

44. Yong GK, Mi JK, Lim JS, Lee MS, Kim JS, Yoo YD. ICAM-3-induced cancer cell proliferation through the PI3K/Akt pathway. Cancer Lett. 2006;239(1):0-110.

45. Park JK, Park SH, So K, Bae IH, Um HD. ICAM-3 enhances the migratory and invasive potential of human non-small cell lung cancer cells by inducing MMP-2 and MMP-9 via Akt and CREB. Int J Oncol. 2010;36(1):181-92.

\section{Publisher's Note}

Springer Nature remains neutral with regard to jurisdictional claims in published maps and institutional affiliations.
Ready to submit your research? Choose BMC and benefit from:

- fast, convenient online submission

- thorough peer review by experienced researchers in your field

- rapid publication on acceptance

- support for research data, including large and complex data types

- gold Open Access which fosters wider collaboration and increased citations

- maximum visibility for your research: over $100 \mathrm{M}$ website views per year

At $\mathrm{BMC}$, research is always in progress.

Learn more biomedcentral.com/submissions 